\title{
Applied Research on Combination of Real-time Farmland Data Acquisition System and Crop Model
}

\author{
Sun Kaimeng \\ Agricultural Information Institute, Chinese Academy of Agricultural Sciences/Key Laboratory of \\ Agri-information Service Technology, Ministry of Agriculture, Beijing,China 100081 \\ email:sunkaimeng@caas.cn
}

Keywords: Internet of Things (IOT), Data Acquisition, Crop Models

\begin{abstract}
According to the study of the practice, description has been given to the crop simulation model and its data acquisition situation, the composition of real-time farmland data acquisition system, as well as the combining point of using both, focusing on the impact of real-time farmland data acquisition system on crop model research, with the significance and value of this change summed up, and the future research discussed.
\end{abstract}

\section{- Crop Model and Its Data Acquisition}

Crop model, also known as crop growth simulation model, is a computer simulation program which gives a quantitative and dynamic description of crop growth, development and yield formation processes and their response to environment[1].The crop model takes a systematic and scientific point of view, sets up environment driving variables including light, temperature, water, soil and other conditions, and applies mathematical physics methods and computer technology, to quantitatively describe and predict photosynthesis, respiration, transpiration and other important physiological and ecological processes during crop growing period as well as their relationship to environmental conditions such as weather, soil, etc. and technical conditions such as farming, irrigation, fertilization, etc., so as to reproduce crop growth and yield formation[2]. With the development of computer technology, crop growth simulation has seen a great development.

Crop model is a mathematical model using computer technology for the simulation of crop growth process, which requires a lot of data as input to the model. Our research team has been devoted to the research and system development of wheat and corn crop model since 1980s; we divide the input variables of crop modelinto three main categories: meteorological data, soil data and crop varieties data. Without doubt, in the application of crop model, there are also experience-based management data, which is not a focus to be discussed in this article. The above-mentioned three types of data are the basis for crop simulation; without the support of these data, the simulation process cannot be completed.

The research and system development process show that the previous crop model data acquisition was all historical data-based, with accuracy and timeliness problems. For example, it is very difficult to get soil data; therefore we can only simplify it by estimating soil moisture variables through relative soil moisture humidity. The stratified soil data of wheat and corn models are basically not available. As we all know, the number and accuracy of input data have a direct impact on crop simulation model, also on the application of crop models. Therefore, input data is the key for crop simulation results whether they are correct or not, and available or not.

\section{- Iot Technology and Real-Time Farmland Data Acquisition}

In recent years, the rapid development of information technology can be witnessed in agriculture, including 3S technology, IoT technology and other new information technologies quickly penetrating into the agricultural sector[3]. IoT technology basically integrates sensor technology and modern communication technology, combined with cloud computing technology, to form a wireless sensor network for remote data acquisition and data exchange, which is important for the 
production environment data acquisition of crops living in the natural environment, as it makes remote acquisition of agricultural environmental data and intelligent management and monitoring of production possible.

There have been defects in collecting crop production environmental data over the years that the meteorological data depend on meteorological department, while crop pattern and soil data depend on manual measurement, with data lag and methods too simple to guarantee the accuracy of data, not to mention data timeliness; also the frequency of data acquisition cannot be guaranteed.

As IoT technology enters into the field of agriculture, it provides an effective technical means for the remote acquisition of farmland crop growth patterns and environmental data. Based on the need of crop simulation model, we have designed a farmland data acquisition device and its corresponding software management system integrated by data acquisition, remote transmission and dynamic growth monitoring, and applied the dynamically-collected crop growth environmental data to crop model research through many years of studying data acquisition methods, acquisition technology and testing, to find new methods and new means of crop model research, system development and application, with certain progress achieved.

The initial application of IoT in agricultural remote monitoring mostly concentrated in the greenhouse, within a relatively closed environment, which has relatively low requirements for sensors, data transmission and production equipment control[4]. Farmland crop production environment means higher requirements for acquisition parts, transmission equipment and power supply equipment in the natural environment. After several years of experiments, field acquisition equipment is able to collect a number of basic data needed by crop simulation models, although the work of acquisition parts is not stable enough and needs improving, it does make some changes in our crop simulation study.

\section{- The Composition of Real-Time Farmland Data acquisition System}

The farmland data acquisition system consists of three major components:

\section{- Acquisition Parts}

Acquisition parts consist of various sensors. In the field acquisition equipment designed by us, the index data that can be collected and directly used in crop simulation models are: weather data: including maximum and minimum air temperature, precipitation, radiation intensity; soil data: including soil temperature, soil stratified humidity. Acquisition devices can also collect other indicators such as carbon dioxide, UV intensity and wind speed, as well as the remotely-controlled camera of dynamic and static images. It should be particularly noted that the soil data acquisition applies soil moisture hierarchical design, and arranges a soil moisture sensor every $10 \mathrm{~cm}$ in accordance with the needs of the crop model for real-time acquisition of soil stratified humidity, which was definitely unable to get in the past.

- Transmission System

The transmission system consists of storing and wireless communication components. Its function is temporary on-site storage of the collected sensor data, and transfer of these data to the remote user server through the wireless communication network and dump. Transmission modes are classified according to the nature of the data transfer, so as to save communications costs. Text data applies 2G communications, i.e. the SMS transmission. Static and dynamic images are currently using $3 \mathrm{G}$ communication transmission.

- Software Management System

The software management system includes data format conversion (converting data into a file that can be read by crop models), acquisition device control (remote control of acquisition time and frequency, adjusting the camera angle, etc.), statistical analysis of data and icons output and other function modules.

Without doubt, the acquisition device also includes ancillary equipment such as solar power system, stents, etc. 


\section{. The Combination Brings Changes to Crop Simulation Technology Research}

- making the results of crop simulation produce dynamic changes

Crop models were based on historical data in the past, including historical meteorological data, soil data, fertilization and irrigation empirical data as well as crop varieties data to simulate the whole process of growing a crop as well as the biomass changes of a crop, to further simulate its birth results.

With the establishment of the IoT-based real-time farmland acquisition system, we can use crop growth data to a certain period to simulate crop growth status during this period, and determine the crop growing trend to this period according to the results of this simulation. Thus, through combination with real-time farmland acquisition system, we can promote the development of crop simulation model research and explore new methods of dynamic crop growth simulation and crop growth control.

- analyzing crop growth trend from the morphological point of view through real-time image acquisition

The real-time farmland acquisition system introduces real-time acquisition of dynamic and static images, and is able to carry out a three-dimensional morphology research of crop growth pattern through analysis and computer image processing of the acquired images, to promote new progress in dynamic three-dimensional crop simulation research. Meanwhile, we can analyze crop growth trend, crop pests situation and take appropriate management measures with real-time images.

The acquisition of real-time dynamic images in the field not only promotes in-depth development of crop simulation research, but also brings up another effective tool for crop growth monitoring, which analyzes the future growth trend of remote crop and has an impact on the remote control of crop management measures. Therefore, it is quite an effective technique.

- strengthening the application of simulation results of crop models

The previous crop simulation results are based on the entire growing season of a crop for a simulation, so the simulation results and appropriate management measures produced, such as fertilization and irrigation schemes, are based on a crop cycle. With the application of IoT technology-based real-time farmland acquisition system, the growth conditions of a crop growing to a certain stage can be simulated, and management measures can be designed according to crop growing conditions by analyzing the crop growth trend during a certain period, so that timeliness in management measures release can be achieved.

\section{- Conclusion}

The main goal of the crop model is to analyze the soil-crop-environment process and interactions through a model[5]. Although our previous studies have simplified the quantitative description of crop model to some extent, many empirical methods were used in the description of the process, and the acquisition of data is still an important factor hampering crop model precision and availability. The introduction of IoT technology-based farmland data acquisition system provides a new method of data acquisition for the study of crop models. It can contribute a real-time and more accurate analysis on the spatial and temporal variability of climate and soil for crop models, promote the development of crop model research and three-dimensional dynamic simulation of the crop, and provide services for the application research of crop growth simulation results. Therefore, the application of farmland data acquisition system enables the study of crop models better contribute to precision farming services, while making crop model research more targeted, more timely, and the results easier to be applied.

\section{Acknowledgement}

This research was supported by National 863 Programs Funded by Ministry of Science and Technology of China(2013AA102305). 


\section{References}

[1] Xu Xinliang et al, "Advances in Studying RS and GIS Technologies Combined with Crop Models," "Anhui Agricultural Sciences", 2012,40 (16), P 9146- 9150;

[2] Cao Hongxin et al, "Exploring the Development of Crop Models" • "Chinese Agricultural Sciences", 2011,44 (17), P3520-3528;

[3]. Pan Ming, Zhong Feng."Research on the Application of Internet of Things in Modern Agriculture”. Modern Agricultural Equipments, Issue 7 in 2011,P55-56;

[4]. Zhao Xia, Wu Jianqiang,."'Research on the Application of Internet of Things in Modern Agriculture”. Agriculture Network Information, Issue 6 in 2011, P5-8;

[5] Luo Yideng et al, " Problems in the Research and Application of Crop Models", "Agricultural Engineering", 2008,24 (5), P307-312; 